\title{
Driving Electrolyte-Gated Organic Field Effect Transistors with Redox Reactions ${ }^{\dagger}$
}

\author{
Benoit Piro *, Jérémy le Gall, Roberta Brayner, Giorgio Mattana and Vincent Noël \\ Université de Paris, ITODYS, CNRS, F-75006 Paris, France; \\ * Correspondence: piro@u-paris.fr \\ + Presented at the 1st International Electronic Conference on Biosensors, 2-17 November 2020; Available \\ online: https://iecb2020.sciforum.net/.
}

Received: date; Accepted: date; Published: date

\begin{abstract}
Organic Electrochemical Transistors (OECTs) are now well-known, robust and efficient as amplification devices for redox reactions, typically biologically ones. In contrast, Electrolyte-Gated Organic Field Effect Transistors (EGOFETs) have never been described for that kind of application because field-effect transistors are known as capacitive coupled devices, i.e., driven by changes in capacitance at the electrolyte/gate or electrolyte/semiconductor interface. For such kind of transistors, any current flowing at the gate electrode is seen as a drawback. However, we demonstrate in this paper that not only the gate potential can trigger the source-drain current of EGOFETs, which is the generally accepted mode of operation, but that the current flowing at the gate can also be used. balabdyBecause EGOFETs can work directly in water, and as an example of application, we demonstrate the possibility to monitor microalgae photosynthesis through the direct measurement of photosynthetic $\mathrm{O}_{2}$ production within the transistor's electrolyte, thanks to its electroreduction on the EGOFET's gate. This paves the way to the use of EGOFETs for environmental monitoring.
\end{abstract}

Keywords: Electrolyte-Gated Organic Field-Effect Transistor; Cyanobacteria; Photosynthesis; Gate current

\section{Introduction}

Electrolyte-Gated Organic Field-Effect Transistors EGOFETs are original organic transistors for which the dielectric material in-between the gate and the semiconductor, mandatorily present in ISOFETs (Ion-Sensitive OFETs) is absent, so that the electrolyte is in direct contact with the semiconductor. In ISOFETs, the thickness of the dielectric governs the operating potential range of the device. Here, its absence allows particularly low operating potentials, of a few hundreds of millivolts. Another characteristic of EGOFETs compared to ISOFETs is that the gate is not necessarily a pseudo-reference electrode such as $\mathrm{Ag} / \mathrm{AgCl}$; it could be a simple metal wire such as $\mathrm{Ti}, \mathrm{Pt}$ or $\mathrm{Au}$, or even a carbon electrode. The electrolyte may be a solid one, as were designed the first EGOFETs, but could also be a liquid electrolyte such as DI water or current biological buffer such as PBS (phosphate Buffer Saline) [1] and are then operated at mild potentials, avoiding solvent oxidoreduction.

As for conventional FETs, EGOFETs are capacitance-driven devices. In practice, the current flowing through the semiconductor of these devices, called drain current and noted $I_{D}$, is directly proportional to the overall capacitance $C_{G_{-}}$osc between the gate and the semiconductor. This capacitance $C_{G_{-}}$osc comes from the series capacitances of both the gate/electrolyte (G_ELEC) and electrolyte/semiconductor (ELEC_OSC) interfaces, i.e., $1 / C_{G_{-} O S C}=1 / C_{G_{-} E L E C}+1 / C_{E L E C}$ OSC. Because the gate electrode and the OSC are dipping in an electrolyte, they are both subject to electrochemical 
reactions. One is the formation of an electrical double layer (EDL) corresponding to charge reorganization at the interfaces, directly related to the interfacial capacitances $C_{G_{-} E L E C}$ and CELEC_OSC and associated to interfacial potential drops. In short, a small capacitance is associated to a large potential drop while a large capacitance is associated to a small potential drop. It is known that organic semiconductors present EDL capacitances one order of magnitude lower than that of metals. As a consequence, for similar gate and OSC area, the potential drop is higher at the electrolyte/OSC interface and $1 / C_{G_{-} O S C} \approx 1 / C_{E L E C} O S C$, i.e., $C_{G_{-} O S C} \approx C_{E L E C} O S C$. Under this condition, the drain current $I D$ is independent on $C_{G_{-} E L E C}$, i.e., is independent on the gate/electrolyte interface. On the contrary, for small gate areas, the gate/electrolyte interface can be used for biosensing, as demonstrated elsewhere [2-4].

The other reactions expected to occur at the interface are redox reactions, i.e., electron transfers, which then produce a gate current. This explain why EGOFETs are subject to high gate currents $\left(I_{G}\right)$ compared to conventional OFETs. IG comes from oxidation or reduction of the solvent or of dissolved species such as molecular oxygen. We will show here that a gate current leads to a change in voltage drop at the interface, so that a gate current $I_{G}$ can be used to drive the drain current $I_{D}$ of an EGOFET. In other words, an EGOFET can amplify a small gate current into a large drain current. As an example, we show that it is possible to amplify the reduction current of $\mathrm{O}_{2}$ at a $\mathrm{Pt}$ gate into a larger drain current. An application could be the continuous monitoring of photosynthetic organisms present into the electrolyte.

\section{Materials and Methods}

Materials and fabrication procedures were described elsewhere [3,5]. The cyanobacteria Anabaena flos-aquae - Af - (strain ALCP B24) were provided by the National Museum of Natural History in Paris. For cyanobacteria growth, the Bold's basal medium (B3N) was used, into which 2.97 $\mu \mathrm{M}$ of vitamin B1, $1.02 \mathrm{nM}$ of vitamin $\mathrm{H}$ and $0.11 \mathrm{nM}$ of vitamin $\mathrm{B}_{12}$ was added. A Pt microelectrode (diameter $100 \mu \mathrm{m}$ ) was used as gate. Electrical characteristics were acquired with a Keithley 4200 SCS source meter.

\section{Results and Discussion}

As preliminary experiment (not shown), oxygen or argon were alternately bubbled in the transistor's electrolyte while drain and gate currents were monitored, for a gate-source voltage $V_{G S}=$ $-0.8 \mathrm{~V}$ and a drain-source voltage $V_{D S}=-0.6 \mathrm{~V}$. Upon argon bubbling, the gate current decreased, while it increased upon oxygen bubbling. The amplitude of the gate current change was $c a .12 \mathrm{nA}$. We measured a parallel change of the drain current, with an amplitude of ca. $1000 \mathrm{nA}$. This experiment demonstrated that the EGOFET is able to amplify the current of a redox reaction occurring at the gate, into a more intense drain current. In another experiment, $10^{6}$ per $\mathrm{mL}$ Anabaena flos-aquae cyanobacteria were then added into the electrolyte compartment of the transistor (total of $2 \times 10^{5} A f$ in $200 \mu \mathrm{L}$ of $\mathrm{B}_{3 \mathrm{~N}}$ ). As all such photosynthetic organism, $A f$ consumes carbohydrates and oxygen for its respiration, anytime, but produces carbohydrates and $\mathrm{O}_{2}$ from $\mathrm{CO}_{2}$ and $\mathrm{H}_{2} \mathrm{O}$ during the light phase. $\mathrm{O}_{2}$ is not captured inside de cyanobacteria but released into the external medium, where it dissolves, which increases its local concentration. Therefore, the continuous monitoring of oxygen concentration in the medium allows to follow the life cycle of the cyanobacteria over time. Under the same conditions as above, the drain and gate currents were recorded under alternated dark and illuminated periods (Figure 1, left). This result shows that oxygen produced under illumination by the cyanobacteria is reduced at the gate electrode, leading to a gate current then to a drain current increase (in absolute value). Under optimized experimental conditions, the amplitude of the drain current change upon illumination is ca. 70-80 nA while that of the gate is ca. $0.1 \mathrm{nA}$, corresponding to an amplification of two to three orders of magnitude. A control experiment without cyanobacteria in the electrolyte shows no gate current, but a small drain current change upon illumination, of ca. $10 \mathrm{nA}$, which is attributed to the photocurrent produced under semiconductor's illumination (Figure 1, right). 

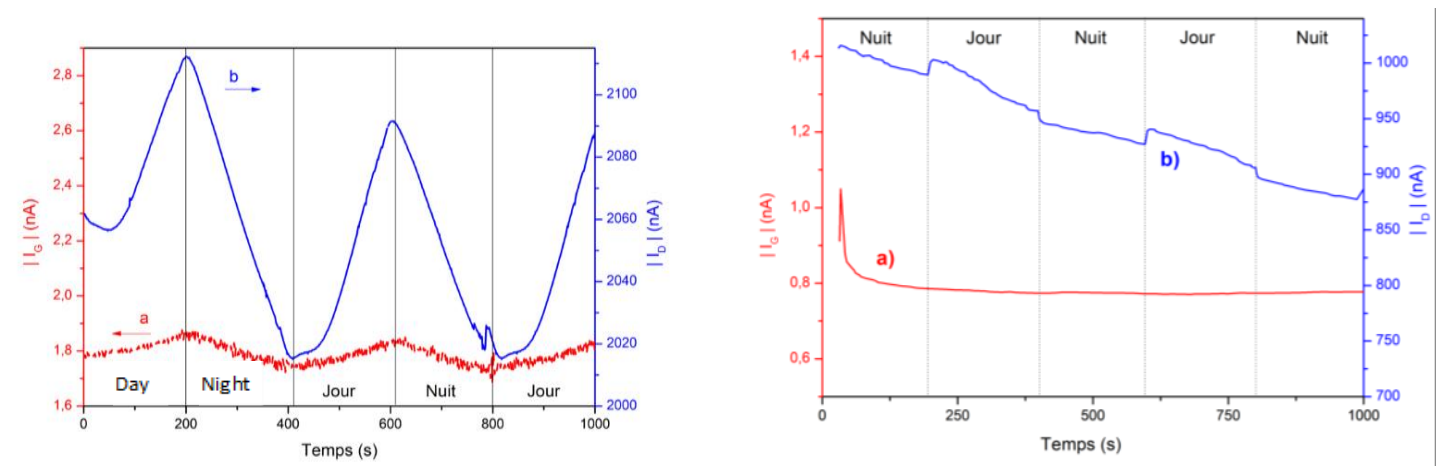

Figure 1. Left, gate current $I_{G}$ (curve a) and drain current $I_{D}$ (curve b) of an EGOFET containing $10^{6}$ cyanobacteria per $\mathrm{mL}$ of electrolyte, under illumination cycles of $200 \mathrm{~s}$ each. Gate diameter $=110 \mu \mathrm{m}$. $V_{G S}=-0.8 \mathrm{~V} ; V_{D S}=-0.6 \mathrm{~V}$. Right, gate and drain current under the same conditions, without cyanobacteria. Only the photocurrent appears on $I_{D}$.

\section{Conclusions}

In this work, we demonstrated that the gate current of an electrolyte-gated organic field-effect transistor is not necessarily a drawback resulting from undesirable electrochemical side-reactions which must be systematically minimized: it can be advantageously used to control the drain current. In other words, an EGOFET is able to amplify up to several orders of magnitude a gate current into a drain current. This is made possible if the gate/electrolyte capacitance $C_{G_{-} E L E C}$ is significantly lower than that of the electrolyte/semiconductor interface, CELEC_OSC, i.e., when the overall capacitance of the EGOFET is driven by $C_{G_{-} E L E C}$ rather than $C_{E L E C}$ OsC. For a given gate voltage, it corresponds to the situation where the potential drop between the gate and the semiconductor is concentrated at the electrolyte/semiconductor interface, maximizing the field effect. We applied this property to the monitoring of the photosynthetic activity of a cyanobacteria (Anabaena flos-aquae) directly added into the electrolyte compartment of the EGOFET. Compared to a regular amperometric $\mathrm{O}_{2}$ sensor, the device is able to amplify the reduction current of several orders of magnitude, typically from a few $\mathrm{nA}$ at a $\varnothing 100 \mu \mathrm{m}$ gate to a few $\mu \mathrm{A}$ at the drain. As an example, we applied the device for monitoring the production of $\mathrm{O}_{2}$ from a cyanobacteria. A perspective could be to use such platform for monitoring the conditions of living organisms in surface waters, i.e., close by industrial areas.

Author Contributions: Conceptualization, B.P.; methodology, B.P., J.l.G., R.B.; investigation, J.l.G.; data curation, B.P., V.N., G.M.; writing, B.P., J.1.G. All authors have read and agreed to the published version of the manuscript.

Funding: ANR (Agence Nationale de la Recherche) and CGI (Commissariat à l'Investissement d'Avenir) are acknowledged for their financial support of this work through Labex SEAM (Science and Engineering for Advanced Materials and devices) ANR-10-LABX-0096, ANR 11 IDEX 0502.

Acknowledgments: JG thanks the Doctorate School ED388 "Chimie Physique et Chimie Analytique de Paris Centre» and Université de Paris for a PhD grant. Are also warmly thank C. Yéprémian and Pr. A. Couté from the Museum National d'Histoire Naturelle de Paris for the cyanobacteria.

Conflicts of Interest: The authors declare no conflict of interest. The funders had no role in the design of the study; in the collection, analyses, or interpretation of data; in the writing of the manuscript, or in the decision to publish the results. 


\section{References}

1. Kergoat, L.; Piro, B.; Berggren, M.; Horowitz, G.; Pham M.C. Advances in organic transistor-based biosensors: from organic electrochemical transistors to electrolyte-gated organic field-effect transistors. Anal. Bioanal. Chem. 2012, 5, 1813-1826.

2. Kergoat, L.; Herlogsson, L.; Piro, B.; Pham, M.C.; Horowitz, G.; Crispin, X.; Berggren, M. Tuning the threshold voltage in electrolyte-gated organic field-effect transistors, Proc. Nat. Acad. Sci. USA 2012, 109, 8394-8399.

3. Nguyen, T.T.K.; Nguyen, T.N.; Anquetin, G.; Reisberg, S.; Noël, V.; Mattana, G.; Touzeau, J.; Barbault, F.; Pham, M.C.; Piro, B. Triggering the Electrolyte-Gated Organic Field-Effect Transistor output characteristics through gate functionalization using diazonium chemistry: Application to biodetection of 2,4dichlorophenoxyacetic acid. Biosens. Bioelectron. 2018, 113, 32-38.

4. Nguyen, T.T.K.; Tran, H.V.; Vu, T.T.; Reisberg, S.; Noël, V.; Mattana, G.; Pham, M.C.; Piro. B. Peptidemodified electrolyte-gated organic field effect transistor. Application to $\mathrm{Cu}^{2+}$ detection. Biosens. Bioelectron. 2019, 127, 118-125.

5. Tibaldi, A.; Fillaud, L.; Anquetin, G.; Woytasik, M.; Zrig, S.; Piro, B.; Mattana, G.; Noël, V. Electrolyte-gated organic field-effect transistors (EGOFETs) as complementary tools to electrochemistry for the study of surface processes. Electrochem. Commun. 2019, 98, 43-46.

Publisher's Note: MDPI stays neutral with regard to jurisdictional claims in published maps and institutional affiliations.

(C) 2020 by the authors. Licensee MDPI, Basel, Switzerland. This article is an open access article distributed under the terms and conditions of the Creative Commons Attribution (CC BY) license (http://creativecommons.org/licenses/by/4.0/). 